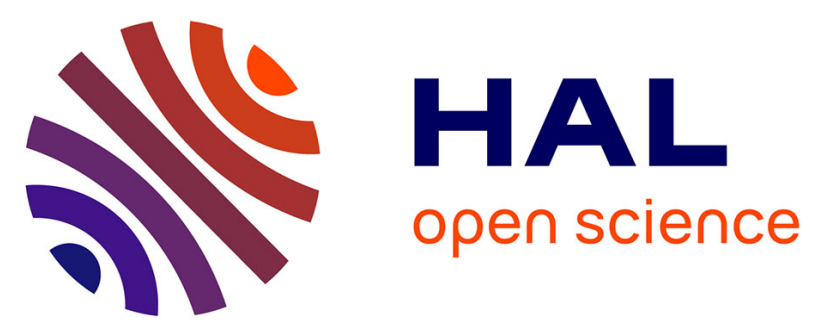

\title{
Estimate of average freeze-out volume in multifragmentation events
}

S. Piantelli, N. Le Neindre, Eric Bonnet, B. Borderie, G. Lanzalone, M. Pârlog, M. F. Rivet, R. Bougault, A. Chbihi, R. Dayras, et al.

\section{- To cite this version:}

S. Piantelli, N. Le Neindre, Eric Bonnet, B. Borderie, G. Lanzalone, et al.. Estimate of average freeze-out volume in multifragmentation events. Physics Letters B, 2005, 627, pp.18-25. 10.1016/j.physletb.2005.08.119 . hal-00008456

\section{HAL Id: hal-00008456 https://hal.science/hal-00008456}

Submitted on 5 Sep 2005

HAL is a multi-disciplinary open access archive for the deposit and dissemination of scientific research documents, whether they are published or not. The documents may come from teaching and research institutions in France or abroad, or from public or private research centers.
L'archive ouverte pluridisciplinaire HAL, est destinée au dépôt et à la diffusion de documents scientifiques de niveau recherche, publiés ou non, émanant des établissements d'enseignement et de recherche français ou étrangers, des laboratoires publics ou privés. 


\title{
Estimate of average freeze-out volume in multifragmentation events
}

\author{
S. Piantelli ${ }^{\mathrm{a}, \mathrm{b}, 1}$, N. Le Neindre ${ }^{\mathrm{a}}$, E. Bonnet ${ }^{\mathrm{a}}$, B. Borderie ${ }^{\mathrm{a}}$, \\ G. Lanzalone ${ }^{\mathrm{a}, \mathrm{c}}$, M. Parlog ${ }^{\mathrm{d}}$, M. F. Rivet ${ }^{\mathrm{a}}$, R. Bougault ${ }^{\mathrm{e}}$, \\ A. Chbihi ${ }^{f}$, R. Dayras ${ }^{g}$, D. Durand ${ }^{e}$, J. D. Frankland ${ }^{f}$, \\ E. Galichet ${ }^{\text {a,h }}$, D. Guinet ${ }^{\mathrm{i}}$, P. Lautesse ${ }^{\mathrm{i}}$, O. Lopez ${ }^{\mathrm{e}}$, \\ E. Rosato ${ }^{j}$, B. Tamain ${ }^{e}$, E. Vient $^{\mathrm{e}}$, M. Vigilante ${ }^{\mathrm{j}}$, C. Volant $^{\mathrm{g}}$ \\ and J. P. Wieleczkof ${ }^{\mathrm{f}}$, \\ INDRA Collaboration \\ ${ }^{\mathrm{a}}$ Institut de Physique Nucléaire, IN2P3-CNRS, F-91406 Orsay Cedex, France \\ ${ }^{\mathrm{b}}$ Dip. di Fisica e Sezione INFN, Università di Firenze, I-50019 Sesto Fiorentino \\ (Fi), Italy \\ ${ }^{\mathrm{c}}$ INFN, Laboratori Nazionali del Sud and Dipartimento di Fisica e Astronomia, \\ via S. Sofia 44, I-95123 Catania, Italy \\ ${ }^{\mathrm{d}}$ National Institute for Physics and Nuclear Engineering, RO-76900 \\ Bucharest-Magurele, Romania \\ ${ }^{\mathrm{e}}$ LPC Caen, IN2P3-CNRS/ENSICAEN et Université, F-14050 Caen Cedex, \\ France \\ ${ }^{\mathrm{f}}$ GANIL, CEA et IN2P3-CNRS, B.P. 55027, F-14076 Caen Cedex 5, France \\ ${ }^{\mathrm{g}}$ DAPNIA/SPhN, CEA/Saclay, F-91191 Gif sur Yvette, France \\ ${ }^{\mathrm{h}}$ Conservatoire National des Arts et Métiers, F-75141 Paris Cedex 03, France \\ ${ }^{\mathrm{i}}$ Institut de Physique Nucléaire, IN2P3-CNRS et Université, F-69622 Villeurbanne \\ Cedex, France \\ jDipartimento di Scienze Fisiche e Sezione INFN, Università di Napoli "Federico \\ II", I-80126 Napoli, Italy
}

\begin{abstract}
An estimate of the average freeze-out volume for multifragmentation events is presented. Values of volumes are obtained by means of a simulation using the experimental charged product partitions measured by the $4 \pi$ multidetector INDRA for ${ }^{129} \mathrm{Xe}$ central collisions on ${ }^{\text {nat }} \mathrm{Sn}$ at $32 \mathrm{AMeV}$ incident energy. The input parameters of the simulation are tuned by means of the comparison between the experimental and simulated velocity (or energy) spectra of particles and fragments.
\end{abstract}

Preprint submitted to Elsevier Science

5 September 2005 
Key words: Multifragmentation, central collisions, freeze-out volume

PACS: 25.70.-z, 25.70.Pq

A better knowledge of multifragmentation properties is of the highest importance in the investigation of the liquid-gas phase transition in hot nuclei $[1,2,3,4]$. In particular, in various statistical and thermodynamical approaches, the concept of freeze-out volume is introduced, which can be defined as the volume occupied by the ejectiles of the multifragmenting source when their mutual nuclear interactions become negligible. Such a volume appears as a key quantity [4] and its knowledge is particularly important in the extraction of fundamental observables such as the microcanonical heat capacity and its negative branch or the shape of caloric curves under external constraints $[1,5,6,7]$.

Up to now volume or density information at freeze-out was derived in various ways. For example by comparing average static and kinetic properties of fragment distributions with statistical multifragmentation models in which the freeze-out volume is an input parameter $[8,9,10,11]$ or from nuclear caloric curves using an expanding Fermi gas hypothesis to extract average nuclear densities [12]. In this work we present a more direct approach to determine freeze-out volumes. Indeed dynamical simulations show that a geometrical picture is fully relevant on the event by event basis and can be used to estimate average volumes of a given class of events [13]. In that context we obtained values of the average freeze-out volume in multifragmentation events, from a "fused system" produced in central collisions, by employing a simulation directly built event by event from the data collected with INDRA [14]. At the present stage we do not want to have a fully consistent understanding of parameter values derived from simulations but rather a very good reproduction of data using reasonable physical hypotheses. Further details of the experimental and calibration procedures may be found in Refs. $[15,16]$.

Complete experimental events (total detected charge $\geq 93 \%$ and total measured momentum $\geq 80 \%$ of the entrance channel values) with flow angle $\geq 60^{\circ}$ (corresponding to compact single source reactions $[17,18,19]$ ) for the reaction ${ }^{129} \mathrm{Xe}+{ }^{\text {nat }} \mathrm{Sn}$ at $32 \mathrm{AMeV}$ were selected. The requested completeness on the total detected charge, more severe than that usually employed by the INDRA collaboration $(\sim 80 \%)[15,16]$, was justified by the necessity of a freeze-out source as close as possible to the reality as input for the simulation, to avoid underestimations of the total Coulomb repulsion among fragments and particles. Main properties of selected multifragmenting sources are summarized in figure 1. Depending on the required completeness on the total detected charge ( $\geq 93 \%$ and $\geq 77 \%$ ), average charged product multiplicity varies from 23.8

$\overline{1}$ Corresponding author: piantell@fi.infn.it 
to 26.2 whereas fragment multiplicity (with charge $\geq 5$ ) increases from 4.13 to 4.72 . One can also verify that the largest completeness does not introduce substantial bias on relevant observables as the differential charge multiplicity distribution, the average experimental velocity of fragments or the width of their velocity spectra. Moreover flow angle distributions for both selections (not shown) exactly superimpose.
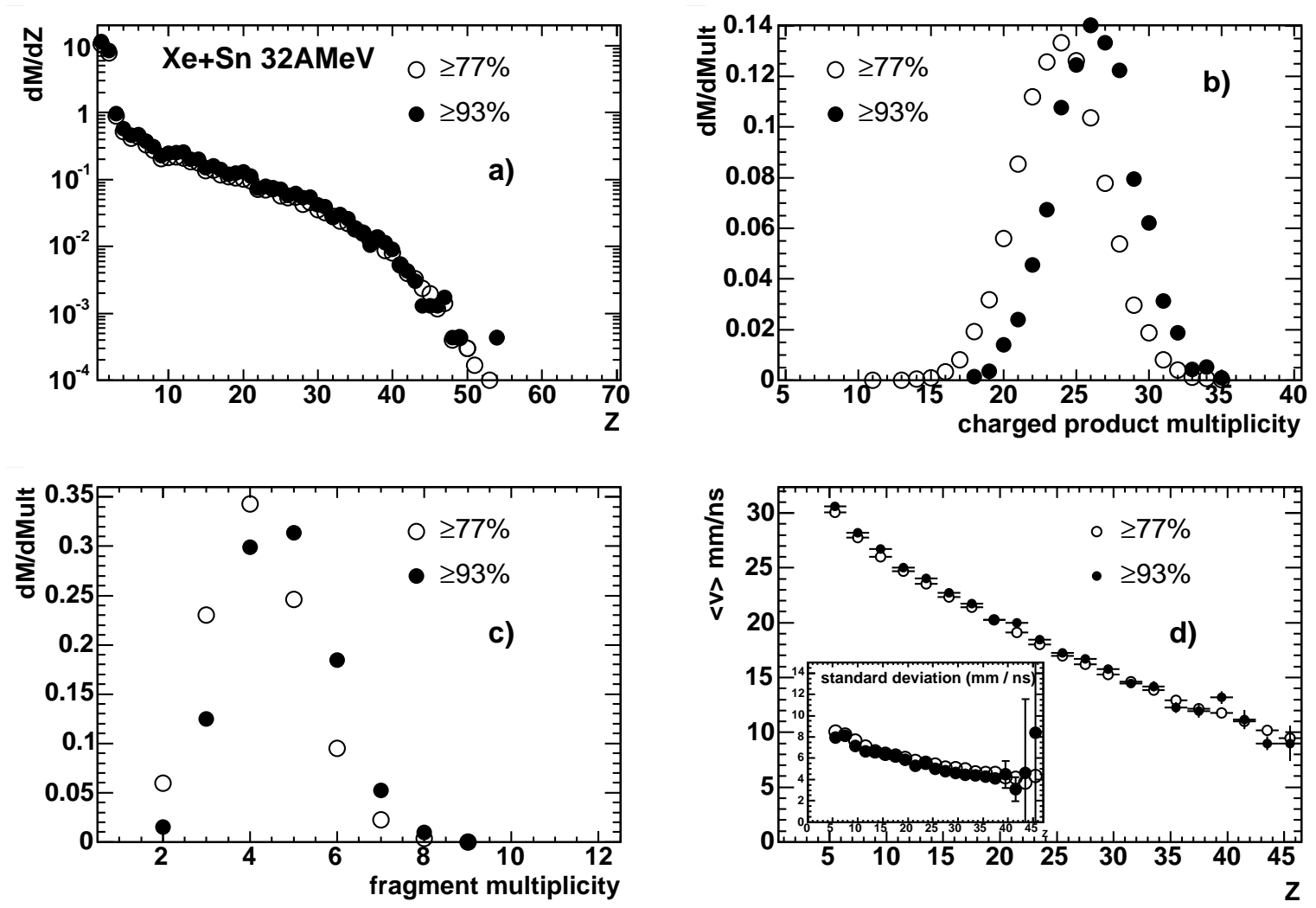

Fig. 1. Properties of selected collisions: (a) differential charge multiplicity distributions, (b-c) total charged product and fragment multiplicities and (d) average and width (standard deviation in the inset) of the centre of mass velocity spectrum of fragments as a function of their charge, regrouped by two charge units. Open (full) symbols correspond to total detected charge greater than or equal to $77 \%$ (93\%) of the entrance channel. Vertical bars are statistical errors.

The multifragmenting source is reconstructed in the reaction centre of mass from all the fragments $(Z \geq 5)$ and twice the particles $(Z=1,2)$ and light fragments $(Z=3,4)$ emitted in the range $60^{\circ}-120^{\circ}$ in order to partially exclude pre-equilibrium emission [20]. Fast isotropic emission can not be removed from the source without any theoretical assumption and, in that respect, calculated excitation energy, mass and freeze-out volume of the source should be considered as upper limits. Atomic mass of detected fragments $(Z \geq 5)$ was calculated from the EAL formula [21]. The number of neutrons (which are undetected) was calculated to keep the $\mathrm{N} / \mathrm{Z}$ ratio of the entrance channel [15]. With such a procedure the average atomic mass and atomic number 
Table 1

Radii for light particles $(\mathrm{H}, \mathrm{He})$ and $r_{0}$ for light fragments, from [22]

\begin{tabular}{cccccccccc}
\hline proton & deuteron & triton & ${ }^{3} \mathrm{He}$ & $\alpha$ & ${ }^{6} \mathrm{He}$ & $\mathrm{Li}$ & $\mathrm{Be}$ & $\mathrm{B}$ & $\mathrm{C}$ \\
\hline $1.03 \mathrm{fm}$ & $2.8 \mathrm{fm}$ & $2.2 \mathrm{fm}$ & $2.4 \mathrm{fm}$ & $2.2 \mathrm{fm}$ & $2.4 \mathrm{fm}$ & $1.7 \mathrm{fm}$ & $1.5 \mathrm{fm}$ & $1.45 \mathrm{fm}$ & $1.3 \mathrm{fm}$ \\
\hline
\end{tabular}

of the reconstructed sources are respectively 217 and 91 . Then, the partition at freeze-out for each event was built by "dressing" detected fragments proportionally to their measured charge (calculated mass for neutron "dressing") with certain percentages of detected (calculated) particles, light fragments and neutrons. Those percentages constitute one parameter of the simulation. The dressed fragments, assumed to be spherical, and the remaining particles and light fragments, if any, were placed in a compact configuration with a minimum distance $D_{\min }$ among the surfaces. It was realized by first putting particles and fragments at random on the surface of a big sphere with volume equal to $30 V_{0}$ ( $V_{0}$ being the volume of the source at normal density) and then moving fragments and particles one by one toward the centre of the sphere by homothetic steps. $D_{\min }$ is another parameter of the simulation. The radius of each fragment was calculated according to the formula $R=r_{0} A^{\frac{1}{3}}$, where $\mathrm{A}$ is the fragment mass and $r_{0}=1.2 \mathrm{fm}$; for light particles (light fragments) experimental radii (deduced $r_{0}$ ) summarized in table 1 were taken. The radius for neutrons was chosen equal to the proton radius.

For each event a consistent calorimetry was made to derive the excitation energy $E_{s}^{*}$ of the corresponding source which undergoes multifragmentation. Thus the partition between internal excitation energy (for fragments) and kinetic energy at freeze-out was determined. To do that a variation of the level density for fragments was introduced. The level density is expected to vanish at high excitation energies [23] and the formalism adopted here is that proposed in [24], where the level density at excitation energy $\epsilon$ is expressed as the Fermi gas level density $\rho^{F G}$ modified by a modulation factor:

$$
\rho(\epsilon)=\rho^{F G}(\epsilon) e^{-\frac{\epsilon}{T_{l i m}}}
$$

This corresponds to introducing an intrinsic temperature for fragments $T_{\text {frag }}$ which verifies

$$
\frac{1}{T_{\text {frag }}}=\frac{3}{2<K^{f o}>}+\frac{1}{T_{l i m}}
$$

where $\left\langle K^{f o}>\right.$ is the average kinetic energy of fragments and particles at freeze-out and $T_{l i m}$ the maximum temperature attainable by fragments. $T_{\text {lim }}$ is a parameter of the simulation. 
Equations used for calorimetry and to derive the sharing between internal excitation energy and kinetic energy on the event by event basis are the following:

$$
\begin{aligned}
& E_{s}^{*}+\Delta B_{s}=\sum_{k=1}^{M_{c p}} K_{c p}^{k}+\Delta B_{c p}+M_{n}^{f o}<K^{f o}>+M_{n}^{\text {evap }} \theta_{\text {frag }}+\Delta B_{n} \\
& E_{s}^{*}+\Delta B_{s}=\left(M^{f o}-1\right)<K^{f o}>+\sum_{k=1}^{n f r a g} a_{k} \theta_{\text {frag }}^{2}+\Delta B_{f o}+V_{\text {Coul }}^{f o}
\end{aligned}
$$

where $\theta_{\text {frag }}$ is equivalent to the temperature $T_{\text {frag }}$ in an ensemble average. $\Delta B, s, K, c p, n$, and $f_{o}$ stand respectively for mass defect, source, kinetic energy, charged products, neutrons and freeze-out. The neutrons evaporated from primary fragments, $M_{n}^{\text {evap }}$, have an average kinetic energy along the deexcitation chain equal to the initial temperature of fragments $\theta_{\text {frag }}$ [25]. An internal excitation energy equal to $\sum_{k=1}^{n f r a g} a_{k} \theta_{\text {frag }}^{2}$ is associated to the fragments at freeze-out, where $a_{k}=\frac{A_{k}}{10} \mathrm{MeV}^{-1}[26], A_{k}$ is the mass of the $k^{\text {th }}$ fragment and $n f r a g$ is the fragment multiplicity at freeze-out. $M^{\text {fo }}$ is the total multiplicity at freeze-out. The total kinetic energy $\left(M^{f o}-1\right)<K^{f o}>$ is shared at random between all the particles and fragments at freeze-out under constraints of conservation laws (linear and angular momentum). $V_{\text {Coul }}^{\text {fo }}$ is the Coulomb energy of the space configuration for freeze-out previously determined. Nuclear interactions between fragments or particles are neglected and we shall see later that this approximation is reasonable. A radial collective expansion energy $E_{R}$ can also be introduced in equations (3) and (4) according to the formula $E_{R}=\sum_{k=1}^{M^{f o}}\left(\frac{r_{k}}{R_{0}}\right)^{2} A_{k} E_{0}$, where $R_{0}$ is the rms of fragment distances to centre at the freeze-out volume, $E_{0}$ the radial expansion energy at $R_{0}$ and $r$ is the distance of the considered particle/fragment of mass $\mathrm{A}$ from the centre of the fragmented source.

Particles and fragments were then propagated under the effect of their mutual Coulomb repulsion; during propagation fragments de-excited, by means of an algorithm largely inspired by the SIMON code $[27,28]$. The main differences concern the tuning of the emission time during the evaporation sequence in order to reproduce the results of theoretical calculations for neutron emission [29] and the constraint that the evaporated particles are inside the list of the particles placed on each fragment at freeze-out. The used emission barriers come from experimental data ([30] for $Z=1,2$ and [31] for $Z=3,4$ ). In this way at the end of the de-excitation phase we obtain secondary charge (and mass) distributions for fragments close to the experimental (calculated) ones; final charges (masses) are recovered, within two charge units (four mass units), for $98 \%$ (85\%) of fragments. Finally experimental angular and energy resolutions were taken into account. 
The last step was the comparison between experimental and simulated spectra both for the energy of the particles and for the velocity of the fragments (average and width) as a function of their charge, in order to tune the four parameters of the simulation $\left(E_{0}, D_{\text {min }}, T_{\text {lim }}\right.$ and the percentages of evaporated particles). We chose to compare the velocity spectra instead of the energy ones because the velocity is less sensitive to the final mass of fragments at the end of the de-excitation process. The explored range for the percentage of evaporated particles was between $0 \%$ and $100 \%$ (no free particles at freezeout); $30 \%$ was suggested by [15]. For $D_{\min }$ we investigated an interval ranging from $0 \mathrm{fm}$ (maximum possible approach without overlap) up to $5 \mathrm{fm}$; for $E_{0}$ we tested from 0 (no collective radial energy) to $1.2 \mathrm{MeV}$. Finally the explored values for $T_{\text {lim }}$ ranged among $6 \mathrm{MeV}$ and $20 \mathrm{MeV}$; in previous studies on the same or similar sample of events $[20,32], T_{l i m}$ values in the range 10 $12 \mathrm{MeV}$ were derived. The limiting temperature influences mainly the width (standard deviation) of the velocity spectra; the percentage of evaporated particles controls all the studied observables (both the standard deviation and the average value of the fragment velocity spectra and also the energy spectra of light particles). The distance among the nuclei surfaces at freezeout and the radial collective energy control mainly the average of the fragment velocity spectra and, more weakly, the particle energy spectra; $D_{\min }$ and $E_{0}$ are correlated, indeed a larger surface distance implies a weaker Coulomb repulsion which can be compensated for by a larger radial kinetic energy.

A $\chi^{2}$ minimization procedure was used to determine the best fit to the data. To reduce the total influence of particles and light fragments but emphasize their high energy tails very sensitive to freeze-out emissions, $\chi^{2}$ was calculated using all the fragment velocity spectra and the Log of particle and light fragment energy spectra. The best agreement, corresponding to a $\chi^{2}=0.953$, was obtained for the following set of parameters: $T_{l i m}=10 \mathrm{MeV}, 90 \%$ of evaporated particles and light fragments, $D_{\min }=2 \mathrm{fm}$ and $E_{0}=0.6 \mathrm{MeV}$. The obtained values for the percentage of evaporated particles are larger than those extracted in [15]; this discrepancy may be due to some intrinsic lack of sensitivity of the method proposed in [15]. Indeed fragment-particle correlations are only fully sensitive when fragments de-excite with a sufficient distance between them and in that sense it is impossible to go back up to the freeze-out configuration. In any case the values presented in [15] constitute a reliable inferior limit for the percentage of evaporated particles. The total average mass of dressed fragments is 208, which corresponds to $96 \%$ of the mass of the source at freeze-out.

Note that with the retained parameters the calorimetry procedure gives an average excitation energy (thermal+collective) $<E_{s}^{*}>=6.7 \mathrm{AMeV}$ for the source which undergoes multifragmentation, which leads, for fragments, to a temperature $T_{\text {frag }}=<\theta_{\text {frag }}>=6.3 \mathrm{MeV}$ and an average excitation energy of 3.9 AMeV. The average kinetic energy of fragments and particles at freeze-out 
corresponds, if interpreted in terms of "kinetic temperature", to a value $T_{\text {kin }}$ of $17.5 \mathrm{MeV}$. A deep understanding of those different numbers and their relation with the observed fragment partitions is out of the scope of the present letter. However we can mention three possible explanations that one will have to discuss in a near future. One is fully related to the procedure followed here, assuming thermal equilibrium for fragments, which reveals the major role of a limiting temperature (for fragments); in that context the influence of $T_{\text {lim }}$ on partitions in microcanonical multifragmentation model like [11] will have to be checked in details. A second one refers to a fast fragmentation for which particles and fragments are early emitted: average primary kinetic energies are then related to the Fermi momentum [33,34,35]. Finally the "kinetic temperature" deduced could also reflect the fact that few very energetic particles emitted during the expansion-thermalization phase [36] significantly increase the average kinetic energy related to $T_{k i n}$.

In figure 2 the experimental centre of mass average velocity of the fragments (full symbols) is compared to the best simulation (open symbols) as a function of the final fragment charge. From the inset it is possible to appreciate the small absolute gap between the experimental data and the simulation. Coulomb repulsion at freeze-out contributes to $\sim 70-80 \%$ of the calculated average velocities. The standard deviation of the fragment centre of mass velocity

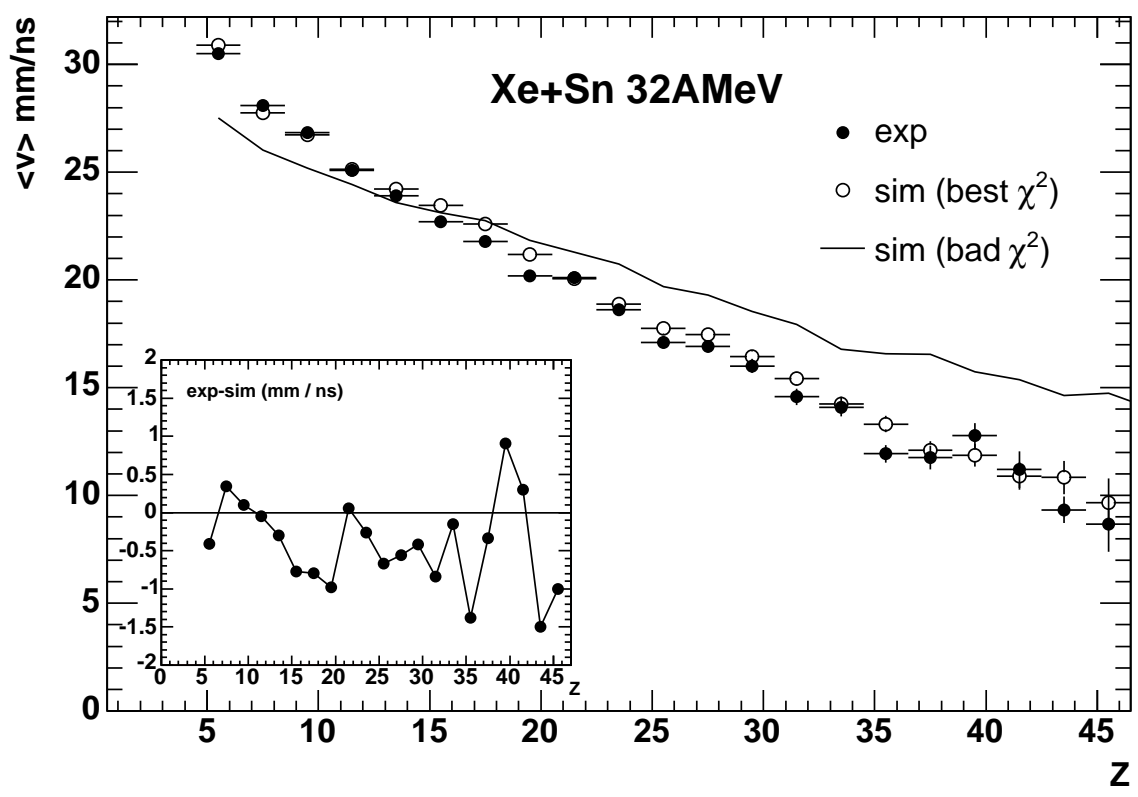

Fig. 2. Experimental (full symbols), best simulated (open symbols) and bad simulated (line, see text) centre of mass average velocity of fragments as a function of the final fragment charge, regrouped by two charge units. Vertical bars are statistical errors. The inset shows the absolute difference between the experimental data and the best simulation. 
spectra as a function of the fragment charge for the experimental data (full symbols) and the best simulation (open symbols) is presented in figure 3. Spatial configurations at freeze-out and fragment decays are only responsible for $\sim 60-70 \%$ of the observed widths and the introduction of a limiting temperature in the simulation turned to be mandatory to account for the experimental values. In figure 4 the experimental and best simulated centre of mass energy

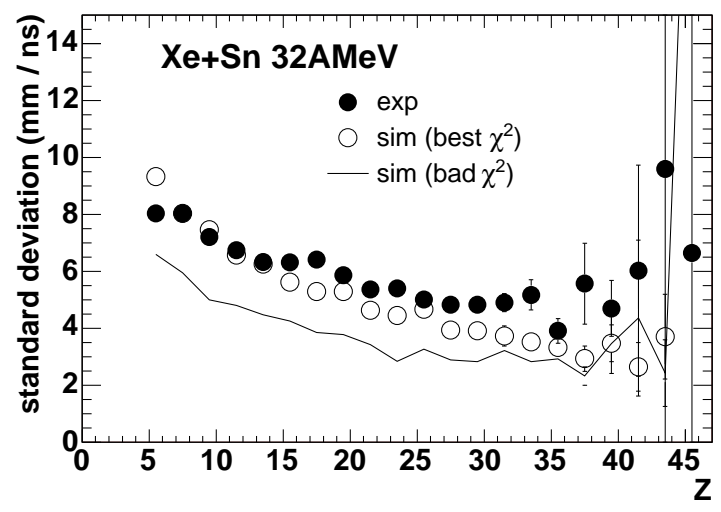

Fig. 3. Standard deviation for the centre of mass velocity spectra of fragments as a function of the fragment secondary charge, regrouped by two charge units (symbols and line as in figure 2). Error bars represent errors coming from the fits.

spectra for particles and lithium fragments are presented. The agreement between simulation and data is good. Relative angles and velocities between fragment pairs were also compared through relative velocity correlation functions (not shown) and exhibit a reasonable agreement between experimental and simulated data.

Before obtaining estimates on freeze-out volume, we can discuss the error bars on the different parameters of the simulation. The standard method was used: errors are extracted from simulations for which $\chi^{2}=$ best $\chi^{2}+1$. The derived ranges for parameters are the following: 9-11 MeV for $T_{\text {lim }}, 70-90 \%$ of evaporated particles and light fragments, $0.5-3 \mathrm{fm}$ for $D_{\min }$ and $0.3-0.6 \mathrm{MeV}$ for $E_{0}$. As previously noted, only those two last parameters are strongly correlated. Therefore, the same reasonable $\chi^{2}$ is obtained for couples of extreme values like $0.5 \mathrm{fm}-0.3 \mathrm{MeV}$ and $2 \mathrm{fm}-0.6 \mathrm{MeV}$ keeping constant $T_{\text {lim }}$ and the percentage of evaporation. Note that even in simulations with $D_{\min }=0.5 \mathrm{fm}$ average distances among the surfaces of nuclei equal to or larger than $2.4 \mathrm{fm}$ are obtained, compatible with the freeze-out definition as the configuration where the nuclear force among the primary products vanishes. Finally to appreciate the sensitivity of parameters, as an example, results from a simulation with $\chi^{2}=$ best $\chi^{2}+3$ are also displayed in figures 2,3 and 4 . For that simulation three parameters have values close to or at the limits of the error bars just discussed before, $T_{\text {lim }}=11 \mathrm{MeV}, D_{\text {min }}=0 \mathrm{fm}$ and $E_{0}=0.34 \mathrm{MeV}$, and one has a value largely outside: $0 \%$ evaporation. It corresponds to a compact freezeout state containing all the detected charged products and calculated number 

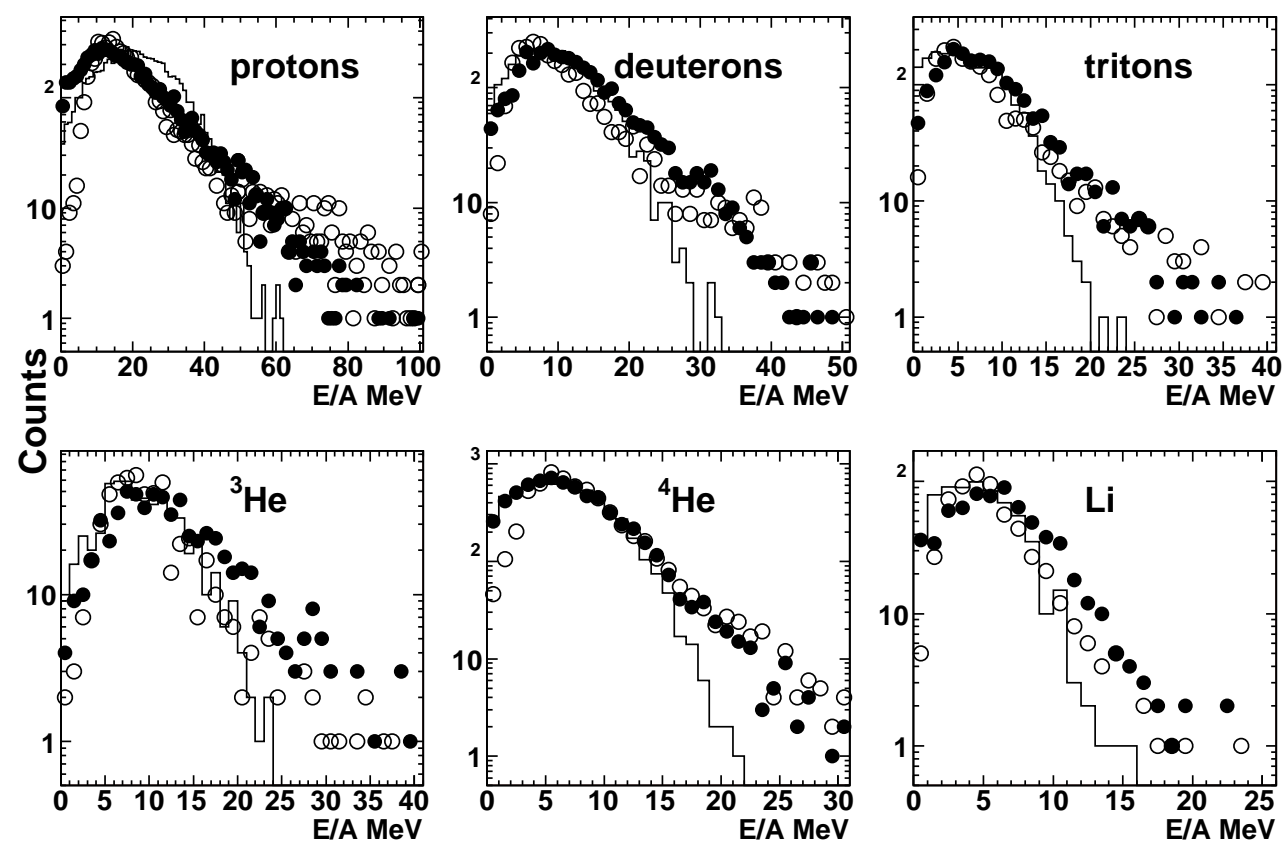

Fig. 4. Center of mass energy spectra per nucleon for light particles and lithium emitted with polar angle in the centre of mass $60^{\circ} \leq \vartheta^{\mathrm{cm}} \leq 120^{\circ}$ (symbols as in figure 2 and histograms for bad $\chi^{2}$ simulation).

of neutrons (sphere volume of $7.4 V_{0}$ - see definition below). We note a wrong slope in the spectrum of the average velocities of fragments versus their charge (figure 2) mainly related to the freeze-out topology imposed by the large number of particles (the heavier the fragment, the larger its distance relative to the center of the volume). The width of the velocity spectra are underestimated (figure 3) due to the absence of smearing effect from evaporation. The energy spectra of particles and light fragments (figure 4) have steeper slopes than the experimental ones because of the absence of evaporation (no boost from primary fragments).

Once the values of parameters and their range defined, the corresponding freeze-out volumes were calculated from the envelopes of nuclei at freeze-out (before starting the Coulomb propagation). The envelope was defined in such a way that two external adjoining spherical nuclei $A_{i}$ and $A_{j}$ (whose centres are located at distances $d_{i}$ and $d_{j}$ from the centre of the fragmenting source) are linked by a portion of sphere with same centre but a radius $R_{e}=1 / 2$ $\left(d_{i}+d_{j}+r_{0} A_{i}^{1 / 3}+r_{0} A_{j}^{1 / 3}\right)$. It is worthwhile to recall that the volume is influenced by two parameters of the simulation: the minimum surface distance $D_{\min }$ and the percentage of evaporated particles, and by the compact configuration built at random. The technique used to calculate the volume of the envelope was to fill up uniformly with points a sphere including all the nuclei and to calculate the ratio among the number of points included inside the envelope and the total number of points inside the sphere. In this way it 
is possible to obtain a good representation of the effective volume occupied by the nuclei also for non-spherical configurations. For the best simulation the average envelope volume is $4.2 V_{0}$; the volume of the sphere including all the nuclei is an overestimation and, on average, for the best simulation, it is $7.65 V_{0}$. Volume estimates, taking into account error bars on parameters, are presented in figure 5. Envelope volumes range from 3.2 to $5.2 V_{0}$ and sphere volumes from 5.7 to $9.6 V_{0}$. A small increase $(\sim 10 \%)$ of envelope volumes is observed when the fragment multiplicity increases from 2 to 8 whereas sphere volumes keep constant whatever the multiplicity. Standard deviations of envelope volumes generated by the simulation realized event by event are close to $10 \%$ of average volumes, which qualitatively agree with predictions, in the coexistence region, of a microcanonical lattice gas model with a constrained average volume $[7,37]$.

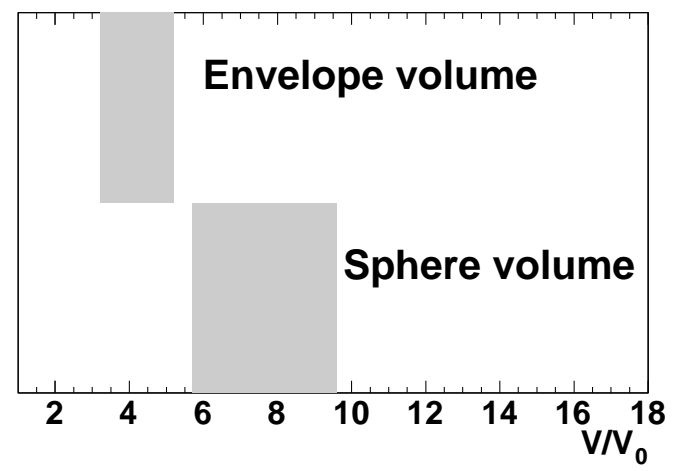

Fig. 5. Estimated average freeze-out volume in units of $V_{0}$. Envelope volumes refer to realistic volumes (see text) and sphere volumes correspond to spheres including all the nuclei at freeze-out.

To summarize, with the powerful $4 \pi$ multidetector INDRA, we were able to obtain information on the freeze-out volume in single source multifragmentation events in the framework of a simulation using the experimental data. The presented method needs data with a very high degree of completeness, which is crucial for a good estimate of Coulomb energy. The use of the widths of fragment velocity spectra in the comparison between data and simulation shows that the introduction of a limiting temperature in the range 9-11 MeV seems mandatory. Work is in progress to study the evolution up to $50 \mathrm{AMeV}$ incident energy of freeze-out volume for the same system and to derive consistent and reliable information from parameters of simulations [38].

\section{References}

[1] D. H. E. Gross, Phys. Rep. 279 (1997) 119. 
[2] J. Richert and P. Wagner, Phys. Rep. 350 (2001) 1.

[3] S. Das Gupta et al., Adv. Nucl. Phys. Vol. 26, edited by J. W. Negele and E. Vogt (2001) p. 89.

[4] B. Borderie, J. Phys. G: Nucl. Part. Phys. 28 (2002) R21\%.

[5] M. D'Agostino et al., Phys. Lett. B 473 (2000) 219.

[6] M. D'Agostino et al., Nucl. Phys. A 699 (2002) 795.

[7] P. Chomaz et al., Phys. Rev. Lett. 85 (2000) $358 \%$.

[8] J. Bondorf, A. S. Botvina et al., Phys. Rep. 257 (1995) 133.

[9] M. D'Agostino, A. S. Botvina et al., Phys. Lett. B 371 (1996) 175.

[10] P. Désesquelles, M. D'Agostino et al., Nucl. Phys. A 633 (1998) $54 \%$.

[11] Al. H. Raduta and Ad. R. Raduta, Phys. Rev. C 65 (2002) 054610.

[12] J. B. Natowitz et al., Phys. Rev. C 66 (2002) 031601(R).

[13] M. Parlog et al., nucl-ex/0503004 and Eur. Phys. J. A in press

[14] J. Pouthas et al., Nucl. Instr. and Meth. A 357 (1995) 418.

[15] S. Hudan et al., Phys. Rev. C 67 (2003) 064613.

[16] G. Tabacaru et al., Eur. Phys. J. A 18 (2003) 103.

[17] J.F. Lecolley et al., Phys. Lett. B 325 (1994) 317.

[18] N. Marie et al., Phys. Lett. B 391 (1997) 15.

[19] J.D. Frankland et al., Nucl. Phys. A 689 (2001) 905.

[20] J.D. Frankland et al., Nucl. Phys. A 689 (2001) 940.

[21] R.J. Charity, Phys, Rev. C 58 (1998) 1073.

[22] Landolt-Bornstein, Numerical Data and Functional Relationships in Science and Technology, Vol. I, edited by A.M. Hellwege and K.H. Hellwege, SpringerVerlag Berlin-Gottingen-Heidelberg (1961).

[23] D.R. Dean et al., Z. Phys. A 322 (1985) $64 \%$.

[24] S.E. Koonin et al., Nucl. Phys. A 474 (1987) 173.

[25] M. Gonin et al., Phys. Lett. B 217 (1989) 406.

[26] S. Shlomo and J. B. Natowitz, Phys. Rev. C 44 (1991) 2878.

[27] D. Durand et al., Nucl. Phys. A 541 (1992) 266.

[28] A.D. N'guyen, Ph.D. thesis, Université de Caen, France, 1998, LPCC T 98-02.

[29] B.Borderie, Ann. Phys. Fr. 17 (1992) 349. 
[30] M.F. Rivet et al., Phys. Rev. C 25 (1982) 2430.

[31] L. Vaz et al., Z. Phys. A 311 (1983) 89.

[32] G. Lanzalone et al., Proc. Int. Workshop on Multifragmentation and Related Topics IWM2001, Catania, Italy, edited by G. Agnello, A. Pagano and S. Pirrone, Laboratori Nazionali del Sud and Sezione di Catania (INFN) p. 154.

[33] A. S. Goldhaber, Phys. Lett. B 53 (1974) 306.

[34] J. Lukasik et al., Phys. Lett. B 566 (2003) 76.

[35] D. Lacroix et al., Phys. Rev. C 69 (2004) 054604.

[36] W. A. Friedman, Phys. Rev. C 42 (1990) 667.

[37] F. Gulminelli, private communication

[38] S. Piantelli et al., to be published 